\title{
HOW SHOULD FAULTS BE NAMED AND CLASSIFIED?
}

Sir:-The interesting discussion which has been carried on recently through the medium of your journal has developed very clearly that the complicated phenomena of faulting do not lend themselves readily to a simple classification. Among other things it has been explained how normal and reversed faulting may take place simultaneously and how faulting due to compression may be erroneously interpreted as normal by neglecting the horizontal component of the fault movement. Emphasis has been laid upon the fundamental difference between faulting caused by igneous intrusion and super-elevation, and that due to compression.

I think that all will admit that the difficulties of the subject impede the adoption of a satisfactory classification, but were the subject simplicity itself, I do not believe that it can be mastered as long as we think of faulting in two dimensions instead of three. A line is a rather narrow representation of a fault plane; and while we may agree with Spurr that the movement may be in any direction on the fault plane, any direction becomes either up or down just so long as the plane is represented either graphically or mentally by a line. It would seem then that before we can classify faults, we must pay some attention to the classification of fault movements.

We may be able to make the complete measurements, viz., the amount and direction of movement on the fault plane-or perhaps only the vertical or horizontal component can be determined - or again the components perpendicular and parallel to the dislocated formation. All these measurements should have names. Again we may measure merely the distance the two parts of a broken formation have been separated and we may measure this either perpendicularly to the stratum or vertically. We may detect and measure faulting as shown in a section, without being able to determine the movement on the fault plane. Lastly we want names for the shifting of the strata as shown in a horizontal plane (the earth's surface). 
It might seem unnecessary to call attention to the various functions of fault movement as they have been so thoroughly explained by Spurr, but I offer as excuse the fact that detailed underground mapping has forced upon me an appreciation of the importance of getting a stereogrammatic conception of fault movement. Spurr's names have priority and the best of authority behind them, but they are neither complete or easy to remember. For instance "lateral separation," " perpendicular separation," and "vertical separation" all lie in different planes. In the list I here offer all measurements on the fault plane are called "displacements." Those on a plane normal to both fault plane and faulted stratum are called "separations." Those shown in any vertical section are called "throws," and those on a horizontal plane (the earth's surface) "heaves."

In defining these functions I assume that the movement on the fault plane is pure translation and that the fault plane is really near enough to a plane to be so considered. Where the plane is distorted and the movement complicated, of course it cannot be defined by simple functions.

In an article published last year I stated "Faults are always measured with reference to the displaced body, and not resolved into horizontal and vertical components, as has been the practice heretofore. It is only by finding a displacement of some such regular body that the fault movement can be measured, and for this and other reasons it is convenient to measure the movement in a parallel and perpendicular direction to the reference strata."'1

In detailed underground work I have found such a resolution of the movement is most convenient. Jaggar, however, has insisted that vertical measurements are important in triangulating thicknesses, and as cases doubtless arise where the vertical or horizontal component is of interest, I have included such a series of measurements in my list.

I therefore submit the following list of measurements in the hope that it may lead to a closer investigation of the subject and

1 "Methods of Investigating Problems in Faulting," Mining Magazine, February, 1906. 
help in the development of a three-dimension concept of fault dislocations.

Displacements-These are the measurements taken on the fault plane. Total displacement ( $a-b$, Fig. 6o) is represented by

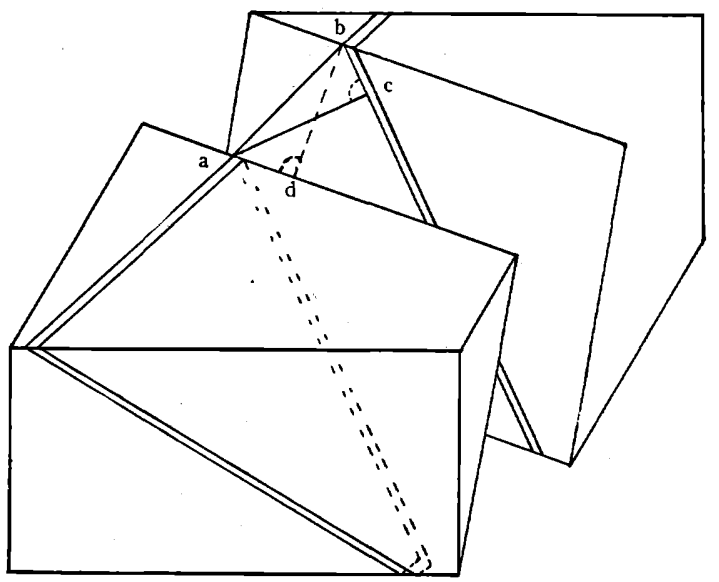

FIG. 6o. Diagram illustrating displacements.

a line connecting portions of a faulted bed originally contiguous but now separated by the fracture. Let this line be the hypotenuse of a right-angled triangle, the opposite side of which is perpendicular to the faulted stratum. The adjacent side of this triangle represents the parallel displacement $(b-c)$ and the opposite side the perpendicular displacement $(a-c)$.

Construct a new triangle, the total displacement again being the hypotenuse and the adjacent side a horizontal line. The adjacent side may then be called the horizontal displacement $(a d)$ and the opposite side $(b-d)$ the vertical displacement.

Separations.-The second set of measurements (Fig. 6I) are in a plane normal to both the fault plane and the faulted bed. The measurement most often taken in practice is the perpendicular distance between the two portions of the faulted formation, or portions produced. This is the perpendicular separation $(c-d$, Fig. 6I). The adjacent side of the triangle $(a d)$ is the parallel separation. The hypotenuse $(a-c)$ is a fault plane measurement, and as such has already been named the perpen. 
dicular displacement. The measurements $a-e$ and $c-e$ may be called respectively horizontal and vertical separation, the two sides of the triangle being parallel and perpendicular to the horizon.

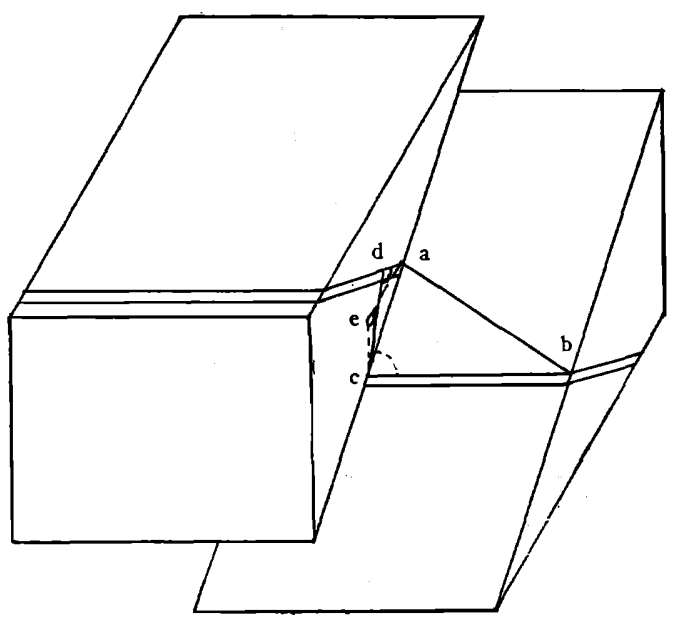

FIG. 6I. Diagram illustrating separations.

Throws.-The throws are measurements taken in any vertical section. The most important section in reference to the faulting is of course at right angles to the strike of the fault. To make the classification perfectly definite I suggest that when the term throw is used, it be understood that the section is normal to the strike of the fault. When the throw in other sections is under consideration the statement should be made that section is not perpendicular to the strike of the fault. As shown in Fig. 62, we have total throw $(a-b)$, perpendicular throw $(a-c)$, and parallel throw $(b-c)$; or again resolving into vertical and horizontal components, we have horizontal throw $(b-d)$ and vertical throw $(a-d)$.

Heaves.-Finally the heaves are the shifts of the strata shown in a horizontal plane and are named in a similar manner. In Fig. $63(a-b)$ is the total heave, $(a-c)$ the perpendicular heave, and $(b-c)$ the parallel heave. Of course there can be no such thing as vertical and horizontal heave. 


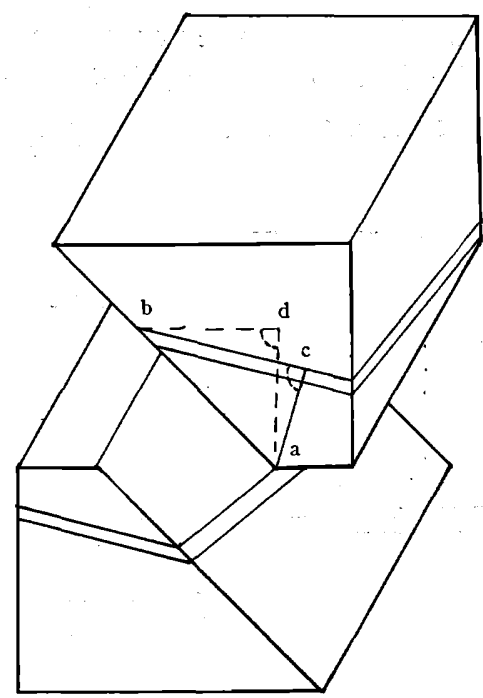

FIG. 62. Diagram illustrating throws.

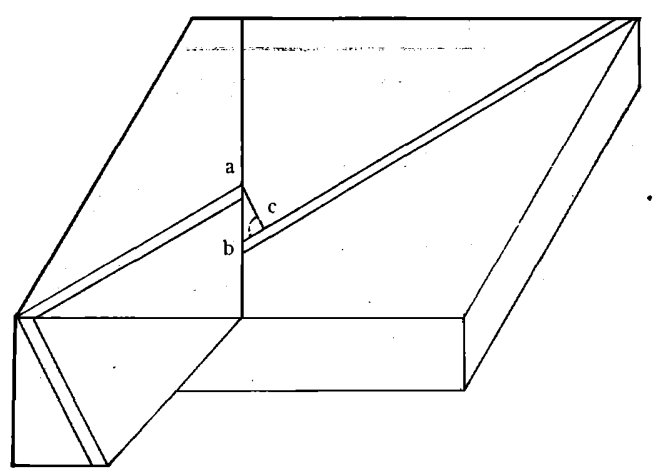

FIG. 63. Diagram illustrating heaves.

Below is a list in which Spurr's names are compared with those here suggested.

\section{SPURR'S NAMES.}

Total displacement,

Lateral separation,

No name,
NAMES SUGgested.

Total displacement.

Perpendicular displacement.

Parallel displacement. 
No name,

No name,

Perpendicular separation,

No name,

No name,

No name,

Throw,

Vertical separation,

No name;

No name,

No name,

No name,

Heave or offset,

No name,

UnIVERSity of Arizona.
Vertical displacement.

Horizontal displacement.

Perpendicular separation.

Parallel separation.

Vertical separation.

Horizontal separation.

Total throw.

Perpendicular throw.

Parallel throw.

Vertical throw.

Horizontal throw.

Total heave.

Perpendicular heave.

Parallel heave.

C. F. Tolman, Jr.

\section{RECENT IMPROVEMENTS IN THE UTILIZATION OF COAL. \\ DISCUSSION OF PAPER BY M. R. CAMPBELL.}

Sir:-Referring to the communication by Mr. M. R. Campbell appearing on page 285 , entitled " Recent Improvements in the Utilization of Coal," it would probably have been better had the title read "The Comparative Heat Efficiency of Gas and Steam Power of the Experiment Plant of the Geological Survey." It is not my object, however, to criticize the title, but to call attention to the fact that we have too much argument relative to the comparative heat efficiencies of the two methods of power development and too little data regarding the cost of producing power by the two methods.

Mr. Campbell says, quoting the first line of his paper-" The better utilization of coal and the cheaper production of power are questions of vital interest to almost every person in the country, for the foundation of modern civilization is power," and yet to date, so far as I am aware, no figures have been offered by the Geological Survey, stating cost of power development. The 